\title{
Análise da Qualidade de Vida e fatores associados dos Acadêmicos da área de saúde de uma Faculdade Particular
}

\author{
Bárbara Oliveira Santos ${ }^{1}$; Felipe Oliveira Bittencourt ${ }^{2}$
}

\begin{abstract}
Resumo: A qualidade de vida dos estudantes vem sendo modificada devido à adaptação ao uma nova realidade. Objetivo: Analisar os fatores associados à qualidade de vida de estudantes da área da saúde de uma faculdade particular de Vitória da Conquista. Metodologia: a pesquisa possui caráter descritivo e exploratório. Em relação à sua natureza, se classifica como qualitativa e quantitativa. A pesquisa foi desenvolvida município de Vitoria da Conquista/BA. A coleta de dados foi realizada na Faculdade Independente do Nordeste. Os participantes do presente estudo foram selecionados por faixa etária de 19 a 25 anos, em 110 estudantes da área da saúde. Resultados e discussões: $O$ estudo apresentou uma prevalência de mulheres $(73 \%)$, solteiros $(54 \%)$, que trabalham (79\%). Em relação à percepção da Qualidade de Vida $(\mathrm{QV})$, os resultados revelam que há uma prevalência global de QV regular (73,6\%). Em relação aos domínios, ocorre uma prevalência de análise regular, no Meio Ambiente (66,4\%), no domínio Psicológico (77,3\%), e no domínio Físico (77,3\%). Nas Relações Sociais observa-se uma análise boa (52,7\%). Na relação entre sexo e qualidade de vida, os homens apontam possuir melhor QV que as mulheres, visto que os seus índices de positividade são bem maiores, além disso, as mulheres assinalaram um alto valor na variável necessidade de melhoria da QV (17,8\%). Conclusões: Esse estudo acaba por ponderar a necessidade de atenção aos aspectos psicológicos e ambientais dos estudantes universitários, com a adoção de medidas que potencializem a QV, evitando impactos negativos tanto na saúde, quanto na vida acadêmica universitária.
\end{abstract}

Palavras-chave: Qualidade de vida. Estudantes. Ensino superior. Estilo de vida.

\section{Life Quality Analysis and Associated Factors of Academic of a Private School Health Area}

\begin{abstract}
The quality of life of students has been modified due to the adaptation to a new reality. Objective: To analyze the factors associated to the quality of life of students in the health area of a private college in Vitória da Conquista. Methodology: the research has a descriptive and exploratory character. In relation to its nature, it is classified as qualitative and quantitative. The research was developed municipality of Vitoria da Conquista / BA. Data collection was done at the Faculdade Independente do Nordeste. The participants of the present study were selected by age group of 19 to 25 years, in 110 students from the health area. Results and discussions: The study had a prevalence of women (73\%), unmarried (54\%), working (79\%). Regarding the perception of Quality of Life (QOL), the results show that there is an overall prevalence of regular QOL (73.6\%). In relation to the domains, there is a prevalence of regular analysis, in the Environment (66.4\%), in the Psychological domain (77.3\%), and in the Physical domain (77.3\%). In Social Relations a good analysis is observed (52.7\%). In the relationship between sex and quality of life, men have a better QoL than women, since their positivity indexes are much higher, in addition, women reported a high value in the variable need for QoL improvement $(17.8 \%)$. Conclusions: This study ends up considering the need for attention to the psychological and environmental aspects of university students, with the adoption of measures that increase QOL, avoiding negative impacts on both health and university academic life.
\end{abstract}

Keywords: Quality of life. Students. Higher education. Lifestyle.

\footnotetext{
${ }^{1}$ Graduanda em Enfermagem pela Faculdade Independente do Nordeste - FAINOR. E-mail: binhalindinha40@ gmail.com;

${ }^{2}$ Orientador, Especialista em Farmácia Hospitalar e Mestre em Saúde e Ambiente. Professor dos Colegiados de Farmácia e Enfermagem FAINOR. E-mail: felipe@fainor.com.br.
} 
Id on Line Revista Multidisciplinar e de Psicologia

Id on Line Multidisciplinary and Psycology Journal

\section{Introdução}

A Qualidade de Vida (QV) sempre se manteve presente na vida do homem, estando associada ao seu interesse em viver de forma saudável e plena. $\mathrm{Na}$ sociedade atual ela é almejada por todos, porém as inúmeras, específicas e subjetivas variáveis para alcança-la são ainda um grande desafio para os profissionais de saúde. A dedicação e busca por uma longevidade não deve ser estabelecida pelos padrões de vida da sociedade moderna mas sim, de uma dedicação e ações de saúde que visem uma boa qualidade de vida, considerando as possibilidades de cada indivíduo (ALMEIDA et al, 2012).

Assim, a qualidade de vida consiste na percepção do indivíduo de sua posição na vida, de acordo com a sua cultura, seus valores, objetivos, padrões de vida, condições físicas e ambientais, relacionamentos e aspectos religiosos e espirituais (WHOQOL, 1995 apud NETTO et al, 2012).

De forma geral a QV é definida por Alves et al (2010), como sendo um conceito de alcance abrangente, afetado de forma complexa pela saúde física, estado psicológico, nível de independência, relações sociais e relações com as características do meio ambiente do indivíduo.

Visto que o conceito de qualidade de vida é considerado usualmente como sinônimo de condição de saúde Cielask et al (2007) enumera que atualmente, há a maior busca por esta condição ideal, ocorrendo a análise dos fatores determinantes da saúde, numa abrangência tanto física quanto mental, visando a melhor compreensão do nível de QV de uma população

Um dos instrumentos validados cientificamente para avaliar a QV é o questionário World Health Organization Quality of Life (WHOQOL), um questionário eu segundo Netto et al (2012) é constituído de questões referentes à aspectos da vida do indivíduo, tais como estado físico e psicológico, relações sociais e meio ambiente.

Dessa forma, a avaliação do nível de qualidade de vida de uma população de estudantes universitários torna-se uma abordagem fundamental, pois como salientam Peckmezovic et al (2011), estes indivíduos estão expostos a fatores bastante complexos em sua vida, durante o processo de escolaridade no nível universitário, sendo esse momento reconhecido como um período particularmente de alta tensão, estando essencialmente relacionadas com as taxas de desgaste e realizações acadêmicas. 
Id on Line Revista Multidisciplinar e de Psicologia

Id on Line Multidisciplinary and Psycology Journal

Segundo Damasceno et al (2015), a entrada do aluno no ensino superior provoca modificações no seu dia-a-dia, permitindo-lhe novas experiências e sentimentos, o que irá influenciar sua percepção sobre a sua qualidade de vida e do seu bem-estar. Para Alves et al (2010), quando o indivíduo não consegue se adaptar à essas mudanças podem ser desencadeados problemas e insucessos com os estudos, podendo levar à um desconforto emocional, que afetará o seu bem-estar.

De acordo Araújo, Soares e Henrique (2009), a competição exacerbada que existe no mundo moderno, além das buscas pelo ter, a intensidade de atividades diárias, o medo de não conseguir o que se deseja, a pressão da sociedade pelo sucesso abstrato, interferem significativamente de forma negativa na vida do indivíduo, promovendo incertezas e inseguranças quanto ao futuro e desestabilizando sua vida. Essas situações são vivenciadas por muitos alunos do ensino superior, favorecendo um desequilíbrio no seu bem-estar e afetando sua saúde.

Dessa forma, diante das dificuldades para o diagnóstico e consequentemente o desenvolvimento de políticas de saúde individuais e coletivas para os alunos do ensino superior, surge a necessidade do desenvolvimento desse estudo e análise específica da temática. $\mathrm{O}$ objetivo dessa pesquisa é analisar o perfil da qualidade de vida dos estudantes da área da saúde de uma faculdade particular de Vitória da Conquista - BA, bem como os seus fatores associados.

\section{Metodologia}

O objetivo deste trabalho é a descrição das características de determinada população ou fenômeno e de abordagem qualitativa e quantitativa, com pesquisa de campo, exploratória com abordagem descritiva.

A pesquisa é baseada na coleta de dados em uma Faculdade Particular de Vitória da Conquista sobre o nível de qualidade de vida dos estudantes da área de saúde. O estudo foi realizado através da aplicação de um instrumento de caracterização sociodemográfica, além do questionário validado de BECK (1996) em estudantes por faixa etária de 19 a 25 anos, a análise 
sendo feita primeiramente através de estatística descritiva dos dados e posteriormente a utilização da análise Kruskal Wallis, para verificar uma possível associação entre as variareis do estudo foi utilizado um nível de significância de $\mathrm{P}=0,05$ e todas as análises foram feitas no software estatístico SPSS 23,0 da IBM.

\section{Resultados e Discussão}

A amostra foi composta por 110 estudantes universitários, apresentando uma prevalência de mulheres (73\%). Em relação ao estado civil, a maioria verificada foi de solteiros (54\%). E quanto a realização de alguma atividade laboral, denota-se que os alunos entrevistados em sua maioria trabalham (79\%). Os outros resultados dessas variáveis, pertencentes ao perfil sociodemográfico verificado nesse estudo, podem ser observados na Tabela 1.

Tabela 1 - Perfil Sociodemográfico dos entrevistados.

\begin{tabular}{lcc}
\hline Variáveis & Frequência & Porcentagem $(\boldsymbol{\%})$ \\
\hline Sexo & 73 & 66,4 \\
Feminino & 37 & 33,6 \\
Masculino & & \\
Estado Civil & 43 & 39,1 \\
Casado & 08 & 07,3 \\
Divorciado & 54 & 49,1 \\
Solteiro & 03 & 02,7 \\
Viúvo & & \\
Que trabalham & 79 & 71,8 \\
Sim & 31 & 28,3 \\
Não & & \\
\hline
\end{tabular}

Fonte: Pesquisa

Os dados do perfil sociodemográfico verificados nesse estudo estão em consonância com os averiguados na maioria dos trabalhos encontrados na literatura, e que versam sobre a qualidade de vida de estudante no ambiente universitário. 
Id on Line Revista Multidisciplinar e de Psicologia

Id on Line Multidisciplinary and Psycology Journal

Em relação ao sexo, vários trabalhos apresentam prevalência de mulheres na amostra de estudantes universitários, dado também verificado nesse estudo. Andrade et al (2011), numa pesquisa com 285 estudantes universitários percebeu que 83,5\% eram mulheres. Alves et al (2010), analisando 370 estudantes, observou que a maioria deles eram mulheres (56,7\%). $\mathrm{Na}$ caracterização sociodemográfica dos participantes do estudo de Paro e Bittencourt (2013), revelou predominância do sexo feminino (75\%) entre 630 graduandos pesquisados.

No entanto, alguns estudos não apresentam uma prevalência do sexo feminino em suas amostras, contrariando o percentual verificado na maioria das literaturas. Petrini, Margato e Junior (2013), numa amostra de 31 estudantes, apontaram a prevalência de 11 sujeitos do gênero feminino e 20 do gênero masculino. Na mesma ótica, Santos et al (2014), também apresentou em seu estudo que a maioria dos estudantes avaliados $(64,16 \% ; n=77)$ era do gênero feminino, um dado estatístico próximo do verificado neste trabalho. Bampi et al (2013), também verificou no perfil epidemiológico dos 84 acadêmicos entrevistados, demonstrou que $57,1 \%$ eram homens e $42,9 \%$ mulheres.

$\mathrm{Na}$ analise do estado civil, a maioria dos trabalhos corroboram com o resultado diagnosticado nesse estudo, onde ocorre uma prevalência de solteiros entre os estudantes da amostra.

Nos estudos de Silva e Heleno (2012), no que se refere às características sociodemográficas dos estudantes, a grande maioria, 93,4\% dos estudantes são solteiros. Alves et al (2010) também verificou essa prevalência com 97\% da amostra composta por solteiros. Santos et al (2014), também notou uma alta ocorrência desse resultado, onde $95 \%$ dos pesquisados se dizem solteiros.

Já em relação ao trabalho, os estudos apresentam resultados diversos não apontando para uma prevalência. Assim em estudos como os de Paro e Bittencourt (2013), averígua-se que a maior parte dos alunos exerce alguma atividade remunerada $(63,1 \%)$, dado semelhante ao evidenciado nesse estudo.

No entanto, em trabalhos como o de Silva e Heleno (2012) aponta-se que 76,7\% da amostra não trabalha, dado também verificado no estudo de Andrade et al (2011), onde 50,35\% da amostra não realiza atividades remuneradas.

Na Tabela 2, estão apresentados os dados referentes a análise da QV, frente as variáveis qualidade de vida, meio ambiente, relações sociais e domínio psicológico. Como resultado, 
Id on Line Revista Multidisciplinar e de Psicologia

Id on Line Multidisciplinary and Psycology Journal

observa-se que na analise da qualidade de vida global, 73,6\% dos pesquisados declaram que a mesma é regular. Em relação aos domínios, nota-se que em quase todos, ocorre uma prevalência de análise regular, sendo que no Meio Ambiente esse índice é 66,4\%, no Domínio Psicológico o índice é de 77,3\%, e no Domínio Físico o índice é de 77,3\%. Somente no domínio Relações Sociais observa-se uma análise positiva, onde o índice é de 52,7\%.

Tabela 2 - Resultado WHOQL dos entrevistados.

\begin{tabular}{|c|c|c|}
\hline Variáveis & Frequência & Porcentagem (\%) \\
\hline \multicolumn{3}{|l|}{ Qualidade de Vida } \\
\hline Boa & 07 & 6,4 \\
\hline Muito Boa & 08 & 7,3 \\
\hline Necessita Melhorar & 14 & 12,7 \\
\hline Regular & 81 & 73,6 \\
\hline \multicolumn{3}{|l|}{ Meio Ambiente } \\
\hline Boa & 21 & 19,1 \\
\hline Muito Boa & 00 & 0,0 \\
\hline Necessita Melhorar & 16 & 14,5 \\
\hline Regular & 73 & 66,4 \\
\hline \multicolumn{3}{|l|}{ Relações Sociais } \\
\hline Boa & 58 & 52,7 \\
\hline Muito Boa & 15 & 13,6 \\
\hline Necessita Melhorar & 05 & 4,5 \\
\hline Regular & 32 & 29,1 \\
\hline \multicolumn{3}{|l|}{ Domínio Psicológico } \\
\hline Boa & 11 & 10,0 \\
\hline Muito Boa & 00 & 0,0 \\
\hline Necessita Melhorar & 14 & 12,7 \\
\hline Regular & 85 & 77,3 \\
\hline \multicolumn{3}{|l|}{ Domínio Físico } \\
\hline Boa & 07 & 6,4 \\
\hline Muito Boa & 00 & 0,0 \\
\hline Necessita Melhorar & 18 & 16,3 \\
\hline Regular & 85 & 77,3 \\
\hline
\end{tabular}

Fonte: Pesquisa 
Id on Line Revista Multidisciplinar e de Psicologia

Id on Line Multidisciplinary and Psycology Journal

Em relação a Qualidade de Vida Geral, a maioria da amostra desse estudo apontou que a mesma é regular, ou seja, não é positiva, mas também não pode ser enquadrada como um fator negativo. No entanto, a maioria dos estudos analisados, apontam resultados diversos aos encontrados nesse trabalho.

Nos estudos de Santos et al (2014), quando questionados sobre como avaliariam sua qualidade de vida, $55 \%$ dos universitários classificaram como boa, 26,66\% como nem ruim nem boa, $13,33 \%$ como muito boa, $5 \%$ como ruim e nenhum como muito ruim.

Netto et al (2012), também averiguou em sua amostra, com relação à pergunta referente à percepção individual da QV, 5,4\% avaliaram-na como ruim ou muito ruim, 19,3\% como nem boa nem ruim, $75,3 \%$ como boa ou muito boa

Meneguci, Valim-Rogatto e Rogatto (2013), também obtiveram resultados mais satisfatórios, já que $83,8 \%$ dos 253 estudantes de educação física avaliados classificaram sua qualidade de vida como boa ou muito boa.

Em relação aos domínios, Meio Ambiente, Domínio Psicológico, Domínio Físico, tal qual na analise da qualidade de vida geral, os resultados dos trabalhos analisados diverge um pouco, sendo que somente no domínio Relações Sociais encontra-se semelhança de resultados.

Catunda e Ruiz (2008), ao analisar a qualidade de vida de universitários, notou uma prevalência de positividade no domínio Físico (80\% da amostra), no domínio Psicológico (77\% da amostra), nas Relações Sociais (77\% da amostra) e no Meio Ambiente (70\%). Em síntese, os resultados do total dos participantes mostram que avaliam como boa sua QVG e que estão satisfeitos com suas condições de saúde, pois os resultados estão acima de $70 \%$ de positividade.

No estudo de Sales e Ferreira (2011), também percebe-se uma prevalência positiva, mas com índices menores apresentando positividade entre os estudantes, sendo no domínio Físico 73,04\%, no domínio Psicológico 68,23\%, nas Relações Sociais 71,67\% e no Meio Ambiente $61,88 \%$

A percepção positiva do domínio físico demonstra que os graduandos pesquisados encontram-se satisfeitos com suas condições físicas, fato que pode estar relacionado com a capacidade íntegra de realização das tarefas cotidianas e corriqueiras dos universitários. Devese considerar que nos dias atuais são muitos aqueles que exercem inúmeras atividades diárias, além dos estudos (MANZATO et al., 2011), para tanto, a habilidade em realizá-las sem que 
Id on Line Revista Multidisciplinar e de Psicologia

Id on Line Multidisciplinary and Psycology Journal

haja algum tipo de desconforto e/ou dificuldade, contribui para a percepção positiva desse domínio.

Em relação ao resultado do domínio das Relações Sociais, propõe-se que o convívio no meio acadêmico estimula a construção de novas amizades e intensifica o convívio social. A promoção da sociabilização no ambiente acadêmico é destacada por Manzatto et al. (2011), indicando que dentro do campo universitário, além do aprendizado, os estudantes buscam por um incremento na vida social.

A percepção relativa ao o domínio psicológico indica que os universitários, , podem estar insatisfeitos com as condições de vida pessoal, fato que, em algum momento da jornada acadêmica, pode refletir sobre a motivação do graduando, pois como destacam Catunda e Ruiz (2008), se o aluno vivencia sentimentos negativos como mau humor, ansiedade e depressão e, além disso não conseguem se concentrar tão bem nos estudos, podem ter cada vez mais prejudicada sua motivação para aprender e seu desempenho.

Deve-se destacar que, normalmente o domínio mais prejudicado e que acaba apontando pior resultado é o meio ambiente, devido a analise, nesse domínio, e facetas relacionadas às condições financeiras e de lazer. No entanto, na contramão da maioria das pesquisas, em que se evidencia essa prevalência, nesse estudo nota-se que o domínio Meio Ambiente foi o que apresentou melhor resultado.

Na Tabela 3, em análise bivariada desse estudo, relaciona as variáveis sexo e qualidade de vida, presentes na amostra de estudantes universitários participantes dessa pesquisa. Os dados coletados dessa associação mostram uma prevalência em ambos os sexos de uma qualidade de vida regular, sendo esse resultado apontado em $67,1 \%$ das mulheres e em $88,6 \%$ dos homens. No entanto, um importante achado nessa analise é que as mulheres apresentam maior evidenciação de necessidade de melhoria da qualidade de vida $(17,8 \%)$.

Tabela 3 - Relação entre Resultado WHOQL de Qualidade de Vida e variável Sexo dos entrevistados.

\begin{tabular}{llrr}
\hline \hline Sexo: & Frequência & Porcentagem \\
\hline Feminino & Boa & 4 & 5,5 \\
\cline { 2 - 4 } & Muito boa & 7 & 9,6 \\
\cline { 2 - 4 } & Necessita melhorar & 13 & 17,8 \\
\hline
\end{tabular}


Id on Line Revista Multidisciplinar e de Psicologia

Id on Line Multidisciplinary and Psycology Journal

\begin{tabular}{llrr}
\hline & Regular & 49 & 67,1 \\
\cline { 2 - 4 } & Total & $\mathbf{7 3}$ & $\mathbf{1 0 0 , 0}$ \\
\hline Masculino & Boa & 3 & 8,1 \\
\cline { 2 - 4 } & Muito boa & 1 & 2,7 \\
\cline { 2 - 4 } & Necessita melhorar & 1 & 2,7 \\
\cline { 2 - 4 } & Regular & 32 & 86,5 \\
\cline { 2 - 4 } & Total & $\mathbf{3 7}$ & $\mathbf{1 0 0 , 0}$ \\
& & &
\end{tabular}

Fonte: Pesquisa

Esses dados mostram que as estudantes mulheres universitárias estão menos contentes com a sua qualidade de vida.

Em consonância com esse resultados Netto et al (2012) verificou que mais estudantes do sexo feminino $(6,2 \%)$ do que do masculino $(3,6 \%)$ avaliaram sua QV como ruim ou muito ruim, dado próximo ao verificado nesse estudo. Nessa mesma ótica, o estudo de Cieslak et al (2012), também demonstrando uma pior avaliação da qualidade de vida por parte das mulheres, verifica-se que, os universitários do sexo masculino demonstraram valores maiores para todos os domínios.

Diante desses resultados, embora não se tenha obtido uma excelência nos resultados dos domínios, a percepção da Qualidade de Vida dos pesquisados é satisfatória, mesmo apontando um resultado diferenciada entre a percepção da QV entre os homens e as mulheres.

Ademais, esses dados indicam uma necessidade de intervenção, pois como ressaltam Cunha e Carrilho (2005 apud PIETRINI, MARGATO e JUNIOR, 2013) o desempenho acadêmico dos graduandos esta relacionado com seu bem-estar físico e psicológico, ou seja, a sua qualidade de vida.

\section{Considerações Finais}

Esse estudo promoveu o diagnostico e análise do perfil da qualidade de vida dos estudantes da área da saúde de uma faculdade particular, bem como os seus fatores associados, 
Id on Line Revista Multidisciplinar e de Psicologia

Id on Line Multidisciplinary and Psycology Journal

a partir dos domínios: Físicos, Psicológicos, Relações Social e Meio Ambiente, relacionandoos com os dados sociodemográficos, que incluem: Sexo, Estado Civil e Trabalha.

$\mathrm{Na}$ analise sociodemográfico no fator sexo, notou-se uma prevalência de mulheres no ambiente universitário. A respeito do Estado Civil, foi identificada a maior parte da amostra é solteira. E quanto a variável trabalho notou-se que a maioria da amostra trabalhava.

Em relação à percepção da Qualidade de Vida, os resultados revelam que há uma percepção satisfatória da QV, com escores positivos nos domínios físicos e sociais. Entretanto, os escores referentes aos meio ambiente são percebidos com maior negatividade. Quanto a Qualidade de Vida Geral, a maioria da amostra apontou que a mesma é regular, discordando de muitos estudos presentes na literatura, em que esse índice é assinalado como bom.

No entanto, na relação entre sexo e qualidade de vida, percebeu-se nesse estudo que os homens apontam possuir melhor qualidade de vida do que as mulheres, visto que os seus índices de positividade para a qualidade de vida geral e seus domínios são bem maiores.

Os resultados obtidos nesse estudo acabam por ponderar a necessidade de atenção aos aspectos psicológicos e ambientais dos estudantes universitários. Em conjunto, enumera-se ainda a necessidade da adoção de medidas que se voltem diretamente a Qualidade de Vida, visando a promoção a integridade e sucesso acadêmico dos universitários, pois a falta de um nível satisfatório de QV pode impactar negativamente sua saúde e gerar dificuldades na vida acadêmica.

Cabe destacar ainda, a necessidade da realização de outras pesquisas, que controlem um maior número de variáveis pertinentes ao conhecimento da QV entre os estudantes universitários e com uma amostra maior, de forma a promover resultados que possam influenciar na elaboração e realização de ações efetivas para essa demanda.

\section{Referências}

ALMEIDA, M. A. B.; et al. Qualidade de vida: definição, conceitos e interfaces com outras áreas, de pesquisa. Revista da Escola de Artes, Ciências e Humanidades - EACH/USP, São Paulo, 2012, p.18. 
Id on Line Revista Multidisciplinar e de Psicologia

Id on Line Multidisciplinary and Psycology Journal

ALVES, et al. Qualidade de vida em estudantes de Medicina no início e final do curso: avaliação pelo Whoqol-bref. Revista Brasileira de Educação Médica, v.34, n.1, p: 91 - 96, Rio de Janeiro, 2010.

ANDRADE, K. O.; et al. Qualidade de vida em estudantes de psicologia. Revista Psicólogo inFormação, ano 15, n. 15, jan./dez. 2011.

ARAÚJO, G. A., SOARES, M. J. G. O. e HENRIQUES, M. E. R. Qualidade de vida: percepção de enfermeiros numa abordagem qualitativa. Revista Eletrônica de Enfermagem, v. 11, n. 3, Goiânia, 2009.

BAMPI, L. N. S.. et al. Qualidade de Vida de Estudantes de Medicina da Universidade de Brasília. Revista Brasileira de Educação Médica, n. 37 (2), 2013.

CATUNDA, M. A. P. e RUIZ, V. M. Qualidade de vida de universitários. Pensamento Plural: Revista Científica do UNIFAE, São João da Boa Vista, v.2, n.1, 2008.

CIELASK, F.; et al. Relação do nível de qualidade de vida e atividade física em acadêmicos de educação física. Revista Fit. Perf. J., n. 6, nov/dez, 2007.

DAMASCENO et al. Fatores Associados à Qualidade de Vida em Estudantes Universitários (Anais). Reunião Anual da SBPC, 67, São Carlos - SP.

DIAS, J. C. R. et al. Qualidade de vida em cem alunos do curso de Medicina de Sorocaba PUC/SP. Revista Brasileira de Educação Médica, v. 34, n. 1, 2010.

MANZATO, L. et al. Consumo de álcool e qualidade de vida em estudantes universitários. Revista da Faculdade de Educação Física da Unicamp, v. 9, n. 1, p. 37-53, jan./abr. 2011.

MENEGUCI, J; VALIM-ROGATTO, P. C; ROGATTO, G. P. Qualidade de vida de estudantes de graduação em Educação Física: comparação entre períodos do curso. Revista digital EFDesportes. Minas Gerais, v.18, n.186, 2013.

NETTO, R. S. M. Nível de Atividade Física e Qualidade de Vida de Estudantes Universitários da área de saúde. Revista Brasileira de Ciências da Saúde, ano 10, nº 34, out/dez 2012.

PARO, C. A.; BITTENCOURT, Z. Z. L. C. Qualidade de Vida de Graduandos da Área da Saúde. Revista Brasileira de Educação Médica, n. 37 (3), 2013

PEKMEZOVIC, T.; et al. Factors associated with health-related quality of life among Belgrade University students. Rev Qual Life Res., n. 20(3), April, 2011.

PIETRINI, A. C.; MARGATO, G. JUNIOR, G. B. V. Avaliação da percepção da qualidade de vida de jovens universitários: comparativo entre graduandos do turno diurno e noturno. Revista Brasileira de Qualidade de Vida, Ponta Grossa - PR, v. 05, n. 03, jul./set. 2013. 
SANTOS, A. K. G. V.; et al. Qualidade de Vida e Alimentação de Estudantes Universitários que moram na região central de São Paulo sem a presença dos pais ou responsáveis. Revista Simbio-Logias, v. 7, n. 10, Dez/2014.

SALES, G. P.; FERREIRA, T. F. Aplicação do Questionário "WHOQOL-BREF" para avaliação da Qualidade de Vida nos participantes do Projeto de Promoção em Saúde Corra pela Vida de São Roque Do Canaã-ES. Revista Brasileira de Prescrição e Fisiologia do Exercício, São Paulo, v.5, n.28, p.366-374. Jul/Ago. 2011.

SILVA, E. C.; HELENO, M. G. V. Qualidade de Vida e Bem-Estar Subjetivo de Estudantes Universitários. Revista Psicologia e Saúde, v. 4, n. 1, jan. - jun. 2012.

SILVA, E. C. Qualidade de vida e bem-estar subjetivo de estudantes universitários. Dissertação (Mestrado). São Bernardo do Campo: Universidade Metodista de São Paulo, 2012.

\section{Como citar este artigo (Formato ABNT):}

SANTOS, B.O.; BITTENCOURT, F.O. Análise da Qualidade de vida e fatores associados dos Acadêmicos da área de Saúde de uma Faculdade Particular. Id on Line Revista Multidisciplinar e de Psicologia, Janeiro de 2017 , vol.10, n.33, p.186-197. ISSN: 1981-1179.

Recebido: $27 / 10 / 2016$

Aceito: $29 / 11 / 2016$ 